\title{
Bechterew, ein Risikofaktor für Herzinfarkt?
}

\author{
„Rheumatiker sterben nicht an ihren \\ Gelenken", titelten wir in MMW \\ Nr.18/2010, S. 14. Eine niederlän- \\ dische Studie scheint dies erneut zu \\ bestätigen.
}

— Niederländische Forscher schickten einen Fragebogen an 593 Patienten mit ankylosierender Spondylitis. Die Rücklaufrate betrug $65 \%$. Von den Respondern gaben $4,4 \%$ an, einen nicht tödlichen Herzinfarkt erlitten zu haben. Dieser Prozentsatz wurde altersadjustiert mit den Daten in der niederländischen Gesamtbevölkerung verglichen.
Hier lag die Zahl bei 1,2\%. Die Autoren meinen daher, dass Morbus Bechterew einen Risikofaktor für Herzinfarkt darstellen könnte.

\section{Kommentar}

Zunächst ist das einmal eine interessante Beobachtung, der man näher auf den Zahn fühlen sollte. Etwas mehr Gewicht erhält sie durch die Tatsache, dass ähnliche Assoziationen zwischen rheumatisch und atherosklerotisch bedingten Erkrankungen schon wiederholt beschrieben wurden. Falls sich der Befund bestätigt, wäre zu fragen, welcher Mechanismus hier zugrunde liegen könnte. Begünstigen vielleicht antirheumatische Medikamente die Infarktgenese? Oder haben rheumatische und atherosklerotische Krankheitsgeschehen einen gemeinsamen Nenner? Für beide Theorien gibt es Anhaltspunkte, jedoch bleiben viele Fragen derzeit noch offen.

E. ERNST

- M. J. Peters et al.

Ankylosing spondylitis: a risk factor for myocardial infarction? Ann. Rheum. Dis. 69 (2010) 579-581

\section{Panperitonitis ohne Bauchschmerzen}

\section{Klagt ein schizophrener Patient über Bauchschmerzen, muss man mit un- angenehmen Überraschungen und Abweichungen von den erlernten Symptommustern rechnen.}

— Eine 51-jährige Frau mit seit dem 40. Lebensjahr bekannter Schizophrenie stellte sich wegen eines seit vier Tagen angeschwollenen Abdomens und Fieber beim Arzt vor. Bei einer rektalen Temperatur von $38,4^{\circ} \mathrm{C}$ war das Abdomen aufgetrieben, jedoch weich und ohne Druckschmerz.

Im abdominellen CT fand man einen großvolumigen gekammerten Aszites, eine Verdickung des Peritoneums im Bereich des kleinen Beckens und im rechten Unterbauch eine teils mit Aszites, teils mit Luft gefüllte Höhle, deren Wand eine Verkalkung aufwies (Abb.). Das Aspirat des Aszites war purulent und wies einen unangenehmen Geruch auf. Kulturell konnten pyogene Erreger, insbesondere Peptostreptococcus und Bacillus subtilis, nachgewiesen werden.

Trotz der unzweifelhaft bestehenden Diagnose einer bakteriellen Panperitonitis war der klinische Zustand der Patientin gut, und sie klagte nicht über
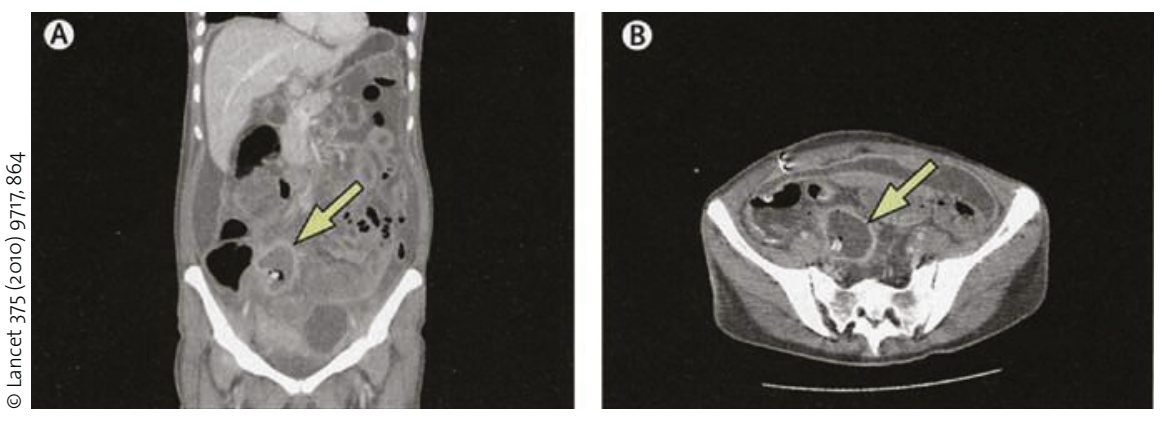

Abdomen-CT: luftgefüllte Höhle mit verkalkter Wand.

Bauchschmerzen. Man versuchte daher zunächst ein konservatives Vorgehen, legte eine Drainage an und behandelte mit Antibiotika. Nachdem sich die Entzündungszeichen nicht besserten, führte man am siebten Tag eine Laparotomie durch, die eine nekrotische und Fäkolithen enthaltende Appendix, große Mengen gelblicher, purulenter Flüssigkeit in der gesamten Bauchhöhle mit einer ausgedehnten Retention im douglasschen Raum ergab. Der postoperative Verlauf war unauffällig und die Patientin erholte sich vollständig.

\section{Kommentar}

Trotz der ausgedehnten Peritonitis wies die Patientin keine Bauchschmerzen, keinen Druckschmerz und keine Abwehr- spannung auf. Sie stand unter einer neuroleptischen Therapie mit Perospiron und Quetiapin. Schizophreniepatienten leiden häufig nicht nur unter Körpersensationen, für die es keine medizinische Erklärung gibt, sie können auch eine abnorme Schmerzverarbeitung von Befunden aufweisen, die bei psychisch gesunden Patienten unweigerlich mit einem akuten $A b$ domen einhergehen. In der Literatur wird über Schizophreniepatienten berichtet, die trotz eines ausgedehnten Myokardinfarkts oder einer Perforation eines gastrointestinalen Hohlorgans weitgehend beschwerdefrei waren. H. S. FüEßL =

- H. Murakami et al.

Altered pain perception in schizophrenia. Lancet 375 (2010) 9717, 864 